\title{
Rolle, Strategien, Aktionsplan 2020
}

In Zeiten dynamischer Neuerungen und Umbrüche sind grundlegende Zielsetzungen, Leitlinien und Konzepte von besonderer Bedeutung. Sie vermitteln Transparenz und Nachvollziehbarkeit des eigenen Handelns. Diese Maximen, die auch bei der Umstrukturierung von Unternehmen und Verwaltungen im Rahmen der Umsetzung der Anforderungen der Datenschutz-Grundverordnung eine maßgebliche Rolle spielten, sind auch für den Landesbeauftragten für den Datenschutz und die Informationsfreiheit (LfDI) wegweisende Grundsätze.

In Zeiten dynamischer Neuerungen und Umbrüche sind grundlegende Zielsetzungen, Leitlinien und Konzepte von besonderer Bedeutung. Sie vermitteln Transparenz und Nachvollziehbarkeit des eigenen Handelns. Diese Maximen, die auch bei der Umstrukturierung von Unternehmen und Verwaltungen im Rahmen der Umsetzung der Anforderungen der DatenschutzGrundverordnung eine maßgebliche Rolle spielten, sind auch für den Landesbeauftragten für den Datenschutz und die Informationsfreiheit (LfDI) wegweisende Grundsätze.

Durch sein Leitbild setzt der Landesbeauftragte die Leitplanken seiner in völliger Unabhängigkeit erfolgenden Tätigkeit und definiert die Rolle, die er bei seiner Aufgabenerfüllung im Gefüge zwischen Wirtschaft, Staat, Justiz und den betroffenen Personen als Berechtigte des Grundrechts auf Datenschutz wahrnimmt.

Die Neuerungen der Datenschutz-Grundverordnung hatten Umstrukturierungen und Anpassungen der Wirtschaft und Verwaltungen zur Folge. Der LfDI leistete und leistet durch zahlreiche Informationsveranstaltungen und die Bereitstellung von Informationsmaterial gezielte Unterstützung der Verantwortlichen und der betroffenen Personen. Die Datenschutz-Grundverordnung hat dem LfDI insbesondere die effektive Durchsetzung des Datenschutzrechts aufgetragen. Welche Strategien und Leitlinien er dabei verfolgt, wie er die Ausübung der zahlreichen, teilweise eingriffsintensiven Befugnisse ausrichtet und welche Früchte die Rechtsdurchsetzung des LfDI bereits getragen hat, werden in dem
Konzept zur effektiven Durchsetzung des Datenschutzrechts in Rheinland-Pfalz zusammengefasst.

Der Aktionsplan enthält Planungen zu Tätigkeiten des LfDI, die Anfang des Jahres 2020 als Zielvorstellungen des LfDI zusammengestellt wurden. Es werden Schwerpunkte der Kontrolltätigkeit, übergreifende Themen, die den LfDI im Jahr 2020 beschäftigen und geplante Veranstaltungen vorgestellt. Aufgrund der Corona-Pandemie ist derzeit nicht absehbar, ob alle Planungen im Jahr 2020 umgesetzt werden können oder teilweise erst im Jahr 2021 realisiert werden. Dies betrifft insbesondere die Untersuchungen und Kontrollen vor Ort und die geplanten Veranstaltungen.

„Es war und ist spannend, die Ausrichtung meiner Behörde, die Strategien und den Fußabdruck, den wir als Datenschutzaufsichtsbehörde in Deutschland und der Europäischen Union hinterlassen wollen, zu gestalten und fortzuentwickeln. Wir freuen uns auf die Herausforderung, weiterhin den Worten Taten folgen zu lassen und uns in den Dienst des Datenschutzes, der Privatheit, sowie der Informationsfreiheit zu stellen und damit unseren Beitrag zur Wahrung der freiheitlichen Demokratie zu leisten.", resümiert der Landesbeauftragte, Prof. Dr. Dieter Kugelmann.

\section{Weitere Informationen}

- Leitbild des Landesbeauftragten für den Datenschutz und die Informationsfreiheit Rheinland-Pfalz: https://www.datenschutz.rlp.de/fileadmin/lfdi/Dokumente/Leitbild.pdf

- Konzept zur effektiven Durchsetzung des Datenschutzrechts in Rheinland-Pfalz: https://www.datenschutz.rlp.de/fileadmin/ lfdi/Dokumente/Konzept_Rechtsdurchsetzung.pdf

- Aktionsplan 2020: https://www.datenschutz.rlp.de/fileadmin/ lfdi/Dokumente/Aktionsplan_2020.pdf

Prof. Dr. Dieter Kugelmann, LfDI Rheinland-Pfalz 